the US National Cholesterol Education Program (NCEP) Third Report of the Expert Panel on Detection, Evaluation, and Treatment of High Blood Cholesterol in Adults (ATP III).

Results After an average follow-up of 6.0 years, the incidence rate of metabolic syndrome among workers who had been working in the same job category for five years was 32.42 per 1000 person-years. Moreover, the largest number of cases with metabolic syndrome appeared in the transportation industry.

Conclusion The results of this study should help the employers of various industries to better plan health education and monitoring programs of their employees to ameliorate the incidence of metabolic syndrome.

\section{P.1.38 EFFECTS OF PARAOXONASE 1 (PON1) GENOTYPE AND NANOMATERIALS EXPOSURE ON HEART RATE VARIABILITY}

Wei-te Wu, Saou-Hsing Liou. National Institute of Environmental Health Sciences, National Health Research Institutes, Miaoli, Taiwan

\subsection{6/OEM-2019-EPI.236}

Background Although paraoxonase 1 (PON1) is a high-density lipoprotein (HDL) associated antioxidant enzyme that has beneficial effects on atherosclerosis and cardiovascular disease, whether PON1 Q192R polymorphism have any effect on cardiac autonomic function is yet to be investigated. Moreover, there is limited data regarding the influence of NPs exposure on HRV parameters in humans. The purpose of the study was to exam whether PON1 genotype or NPs exposure has an association with HRV levels.

Methods This cross-sectional study recruited 235 workers exposed to NM and 185 non-exposed controls from $14 \mathrm{NM}$ handling plants in Taiwan from 2009 to 2011. For each participant, we collected blood specimens to determine the genotype of the PON1 Q192R polymorphism and PON1 paraoxonase and arylesterase activities. In addition, short-term HRV was tested.

Results The results showed that PON1 Q192R genotype and PON1 paraoxonase/arylesterase activities associated with HRV, and was particularly noteworthy in RMSSD and HF. The relationship between NPs exposure and HRV was only found in workers exposed to nano-Ag, but the apparent relationship between NPs exposure and HRV is lacking.

Conclusions It is a novel finding that both RR genotype of PON1 (Q192R) and activities of PON1 increased with HRV levels. PON1 Q192R genotype may play an important role in cardiac protection. Considering the global prevalence of NMs market, further studies of NPs-induced cardiac events and stresses still are required to establish.

\section{P.1.40 FARMER'S SUICIDE WITH THE USE OF PESTICIDES IN DIFFERENT AREAS IN SPAIN}

${ }^{1}$ Guanlan Zhao, ${ }^{1}$ Elena Ronda, ${ }^{2}$ Gregorio Barrio, ${ }^{3}$ Enrique Regidor. ${ }^{1}$ University of Alicante, Alicante, Spain; ${ }^{2}$ National School of Public Health, Instituto de Salud Carlos III, Madrid, Spain; ${ }^{3}$ Complutense University of Madrid, Madrid, Spain

\subsection{6/OEM-2019-EPI.237}

Introduction There is considerable interest in the potential relation of suicide with the pesticide. Agriculture is the leading professional sector for pesticide use in the world with a turnover of about 40 billion US dollars in 2010. According to the WHO report in 2014, autointoxication with agricultural pesticides contributes greatly to the global burden of suicide. The aim of this study is to evaluate whether suicide of farmers was associated with the use of pesticide in different areas during the period 2001-2011 in Spain.

Methods The cohort study followed 9.5 million men in Spain from 2001 to 2011. Participants aged 20-64 years were employed in 2001. The study subjects were grouped as farmers and non-farmers. We divided Spain into two areas depending on both geographical location and the mean annual amount of pesticides used: southeast Spain and the rest of Spain. The southeast Spain shows higher use of pesticides than the rest of Spain. For three major causes of death (suicide, unintentional accidents and rest of causes of death), we estimated the age-standardized mortality rates per 1000,000 person-years of follow-up, the ratio of age-standardized mortality rates with $95 \%$ confidence intervals (CI) between farmers and non-farmers in two areas were calculated.

Results With respect to the rest area of Spain, farmers in the southeast showed a lower mortality rate of suicide and unintentional accidents, which mortality rate ratios were 0.74 (95\% CI $0.65-0.85)$ and 0.57 (95\% CI $0.52-$ $0.62)$.

Conclusion Our findings do no support a possible association between pesticide use and suicide of farmers in Spain.

\section{P.2.01 EPIDEMIOLOGICAL STUDIES OF THE PORTON DOWN VETERANS: AN UPDATE OF CANCER INCIDENCE AND MORTALITY TO 2018}

${ }^{1}$ Gemma Archer, ${ }^{2}$ Thomas Keegan, ${ }^{3}$ Claire Brooks, ${ }^{3}$ Lucy Carpenter, ${ }^{3}$ Katherine Venables, ${ }^{1}$ Nicola Fear. ${ }^{1}$ King's College London, London, UK; ${ }^{2}$ Lancaster University, UK; ${ }^{3}$ Oxford University, UK

\subsection{6/OEM-2019-EPI.238}

Introduction An update to the Porton Down Veterans Cohort study is planned for 2018-2021.

Background Chemical warfare agents continue to be deployed by rogue states and terrorists, e.g. in Syria, Iraq, Tokyo, and Salisbury UK, yet their impact on long-term health is largely unknown. The Porton Down cohort comprises of 18276 men who took part in the 'human volunteer programme' of chemical warfare agent research at Porton Down, UK, between 1941 to 1989 , and a comparison group of 17600 men who also served in the military. An original study linked veterans' records to national registry data on cancer incidence and mortality up to 2004. By the end of 2004, 7306 of the Porton Down veterans and 6900 of the comparison cohort had died. The results showed Porton Down veterans had no overall excess of cancers (rate ratio $(\mathrm{RR})=1.00$, 95\% CI 0.95-1.05) and a small (6\%) excess of all-cause mortality $(\mathrm{RR}=1.06$, 1.03-1.10) when compared with non-Porton Down veterans. Porton Down veterans had higher rates of certain cancers (e.g. ill-defined malignant neoplasms, and in situ neoplasms) and specific causes of death (e.g. genitourinary, circulatory and external causes).

Objectives The updated study aims to replicate the original analyses but with an additional 15 years of follow-up - estimated to increase the number of deaths available for study to c 22 200. This will improve statistical power, allowing 